\title{
Physicochemical properties affecting cellular uptake of carbon nanotubes
}

Carbon nanotubes (CNTs) are widely used for biomedical applications as intracellular transporters of biomolecules owing to their ability to cross cell membranes. In this article, we survey the reported literature and results of our published work in an attempt to provide a rational view of the various CNT internalization mechanisms. Essentially three uptake mechanisms (phagocytosis, diffusion and endocytosis) have been reported in literature. In addressing the subject of cellular internalization of CNTs, the unique physicochemical characteristics of CNTs that influence and drive the cell uptake pathway are considered. According to available evidence, the degree of dispersion, the formation of supramolecular complexes and the nanotube length are crucial factors in determining the exact mechanism of cellular uptake. In conclusion, phagocytosis appears to be the internalization pathway for CNT aggregates, bundles, cluster or single dispersed nanotubes $1 \mu \mathrm{m}$ or more in length; endocytosis is the internalization mechanism for nanotubes forming supramolecular structures; and diffusion is the internalization mechanism for submicron CNTs that do not form supramolecular complexes. This information may be relevant to the rational design of CNT-based carriers for cell therapy.

KEYWORDS: carbon nanotubes internalization mechanisms physical and chemical properties

Carbon nanotubes (CNTs) [1] are molecular-scale tubes of graphite carbon with unique properties including extreme strength and unique electrochemical and other physical characteristics [2]. These properties account for the considerable scientific and industrial interest, as evidenced by the substantial number of original publications on nanotubes reported annually. CNTs are either single-wall CNTs (SWCNTs) consisting of a single graphite lattice rolled into a perfect cylinder or multiwall CNTs (MWCNTs) made up of several concentric cylindrical graphite shells. In the last 10 years, several groups have shown that SWCNTs and MWCNTs can be used as efficient carriers for the delivery of therapeutic and diagnostic small and large molecules inside cells [3-5]. However, in recent years, conflicting data have been reported concerning safety and biocompatibility of these nanotubes [6]. Additionally, the exact mechanism of cellular binding and internalization continues to raise significant debate. There are several reasons for this continuing controversy, chief among which is the lack of characterization of the nanotubes in terms of length and presence of impurities, both intrinsic (e.g., vacancies and charged defects) and extrinsic (e.g., catalyst residue). Some inconsistencies also seem to arise due to differences in experimental protocol, for example the dispersant used to solubilize the nanotubes, type of functionalization, concentration, duration of exposure, method of exposure and the varied in vitro cell assay systems used. Additionally, the lack of standard operating procedures and a centralized toxicity database limit comparison between research results. Another issue affecting the use of functionalized nanotubes for biomedical application is that charge has to be controlled and understood to enable predictable interactions with the biological environment.

In 2003-2006 different groups reported seemingly contradictory data on the internalization mechanisms involved. Pantarotto et al. suggested nanotube uptake by living cells through an energy-independent nonendocytotic pathway that involves insertion and diffusion of nanotubes across cell membranes [7]. A year later, Cherukuri et al. reported active ingestion of the nanotubes by macrophages [8]. An extensive review of the mechanism regulating the cellular internalization of the nanotubes and their cargos was published in 2006 by Shi Kam et al. who reported clathrin-dependent receptor-mediated endocytosis (RME) [9]. These initial reports highlighted the need for alternative approaches for the investigation of the mechanisms involved in the cellular uptake of nanotubes.

Considerable insight into these mechanisms has been obtained in recent years by the use of techniques that have enabled in vitro and in vivo tracking of CNTs. Traditionally, the
Vittoria Raffa ${ }^{1+}$,

Gianni Ciofani',

Orazio Vittorio ${ }^{1,2}$,

Cristina Riggio ${ }^{1}$

\& Alfred Cuschieri ${ }^{1}$

${ }^{\dagger}$ Author for correspondence:

${ }^{1}$ Scuola Superiore Sant'Anna,

Medical Science Laboratory,

56127, Pisa, Italy

Tel.: +39050 995625 ;

Fax: +39050995676 ;

s.raffa@sssup.it

${ }^{2}$ Università degli Studi di Pisa, 56100, Pisa, Italy 
most popular methods for imaging CNTs have included electron microscopy techniques, (e.g., transmission-electron microscopy [TEM] and scanning-electron microscopy) [10,11] and confocal microscopy of nanotubes complexed with fluorescently labeled polymers, quantum dots or silver iodide filling of the nanotubes [12].

The unique properties of the CNTs have only recently been exploited in a variety of molecular imaging applications: Fe-doped or $\mathrm{Gd}^{3+}$-functionalized nanotubes can be detected by MRI and semiconductor nanotubes are readily detected by near-infrared fluorescence. Radionuclide-based imaging (PET and SPECT) have been used to study the biodistribution of radiolabeled nanotubes and Raman spectroscopy and photo-acoustic tomography for CNT imaging in vivo [13].

In this article, the authors have attempted to address the reported conflicting data published since 2003 on cellular uptake of CNTs by scrutinizing the parameters that are likely to influence the cellular internalization process. This analysis of the state-of-the-art highlights that there are three crucial parameters which determine nanotubes interactions with cells: the degree of dispersion (presence of bundles, nanotube clusters or single dispersed CNTs); the formation of supramolecular complexes (complexation with proteins and nucleic acids or adsorption of proteins from extracellular matrix); and nanotube length.

Other parameters, such as nanotube diameter, chirality and number of layers, seem to play a lesser role in the internalization process. In essence, these three parameters affect the mechanism of nanotube internalization as outlined in the following sections.

\section{Strategies for preparation of water soluble nanotubes: $\pi-\pi$ interactions}

The solubility of CNTs in aqueous solvents is essential for biological interactions and biocompatibility; hence, CNT composites for biomedical applications must be rendered water soluble.

Pristine (as-prepared) CNTs aggregate in bundles because of the hydrophobic character of the graphene sidewalls, coupled with the strong $\Pi-\Pi$ interactions between the individual tubes. The crucial role of the CNT dispersion state as a defining factor influencing the cellular uptake of CNTs has often been ignored. Given a constant dosage, differences in dispersion ranging from macroscopic aggregates to micrometer bundles or individually dispersed nanotubes will dramatically affect the absolute size and, hence, the internalization mechanisms.
The most commonly used methods for dispersion (essential for biomedical use) are: surfactant-assisted dispersion, functionalization of CNT sidewalls and biomolecular dispersion (Figure 1) [14,15]. Irrespective of the nature of the dispersion the 'dissolution' process is improved by prolonged sonication, as this provides the mechanical energy necessary to overcome the inter-tube van der Waals forces, resulting in disaggregation into individual CNTs [16]. Another effect of the sonication is reduction of nanotube length by cleavage of CNTs at defective sites.

A variety of anionic, cationic, zwitterionic and nonionic surfactants can be used for CNT dispersion. Dispersion by nonionic surfactants is a function of the hydrophobic alkyl chain length of the surfactant [17]. The high-molecular-weight surfactants and polymers increase the solubility of CNTs through steric stabilization by adsorbed surfactant/polymer on to the walls of the CNTs, thereby impeding aggregation. For ionic surfactant dispersion, Vaisman et al. [18] determined that the controlling factor is the charge of the head group, rather than the hydrophobic alkyl chain length. As the charge ( $\zeta$-potential) increases, the dispersion is stabilized through increased electrostatic repulsion. Some unresolved issues of surfactant-based dispersion include the relatively low levels of solubility and stability of the dispersion.

Various modifications to CNT sidewalls have been made to improve solubility and dispersion in polar solvents by introducing polar functional groups on the CNT surfaces. Covalent functionalizations have employed two general strategies. The first is carboxylation and the subsequent amidation/esterification reactions at the oxidatively introduced carboxyl groups at the defect sites. The second concerns addition reactions. These basically provide a nonselective approach (generally thermal activated, but sometimes plasma or photochemical activated) of CNT sidewalls by highly reactive species such as atoms, radicals, carbenes or nitrenes. Covalent sidewall functionalization is expected to produce the most stable dispersion based on the density of the bound functional groups. The use of biomolecules to disperse CNTs in solution has been described in several reports. CNTs can interact with many biomolecules without forming covalent conjugates. Proteins are an important class of substrates having high affinity with the graphitic network of CNTs. They tend to adsorb strongly on the exterior of nanotube walls presumably via interactions between the graphitic surface and the hydrophobic domains of the biomolecule or even by means of charge-transfer interactions [19,20]. Similarly, 
monoclonal antibodies are known to bind specifically to the surface of nanotubes [21]. Both covalent and noncovalent interactions between DNA and CNTs have been reported. The electrostatic repulsion of the negatively charged phosphate backbone of the DNA, gives rise to a stable CNT dispersion. In the noncovalent approach DNA-CNT conjugates are obtained by sonication with pristine CNTs [9] or cationic CNTs, which are able to condense DNA, for example ammonium-functionalized CNTs $\left(\mathrm{CNT}^{-N H} 3^{+}\right)$, lysine-functionalized CNTs (CNTLys-NH3 ${ }^{+}$) [22], polyethylenimine-graft MWCNTs (PEIgMWCNTs) [23]. In covalent approaches, CNTs are bound to DNA via amide linkages $[24,25]$.

\section{The internalization mechanisms reported in literature: phagocytosis, diffusion \& endocytosis Phagocytosis}

In the experiments reported by Porter et al. [11], SWCNTs were dispersed in tetrahydrofuran by ultrasonication and mixed in the cell culture medium. No dispersing reagent (e.g., surfactant) was used to break up the as-prepared nanotube bundles. These authors observed cellular uptake of SWCNTs by electron microscopy. After 4 days exposure to human monocyte-derived macrophages, the majority of SWCNTs were located within phagosomes and lysosomes of healthy cells, suggesting uptake by phagocytosis. Cherukuri et al. reported that macrophage cells can actively ingest significant quantities of $1 \mu \mathrm{m}$ long SWCNTs [8].

\section{Diffusion}

We have previously demonstrated that Pluronic ${ }^{\circledR}$ F127-coated MWCNTs are internalized by an energy-independent pathway [26]. Pluronic F127 is an amphiphilic block copolymer and its brushlike configuration presents a repellent surface to proteins. Hence, energy-dependent uptake mechanisms such as endocytosis were not involved in these experiments. Endocytosis is a recognized general entry mechanism for various extracellular materials and is an energy-dependent process. It is depressed by low temperature incubations or in ATP-depleted environments [27]. In our experiments, no difference in penetration capacity was observed between 37 and $4^{\circ} \mathrm{C}$ and following treatment of the cells with a metabolic inhibitor, sodium azide $\left(\mathrm{NaN}_{3}\right)$. Our investigations also demonstrate that nanotube length clearly influences uptake and shorter (sub-1 $\mu \mathrm{m})$ MWCNTs are readily internalized by cells. Short nanotubes (i.e., hundreds of nanometers in length) can act

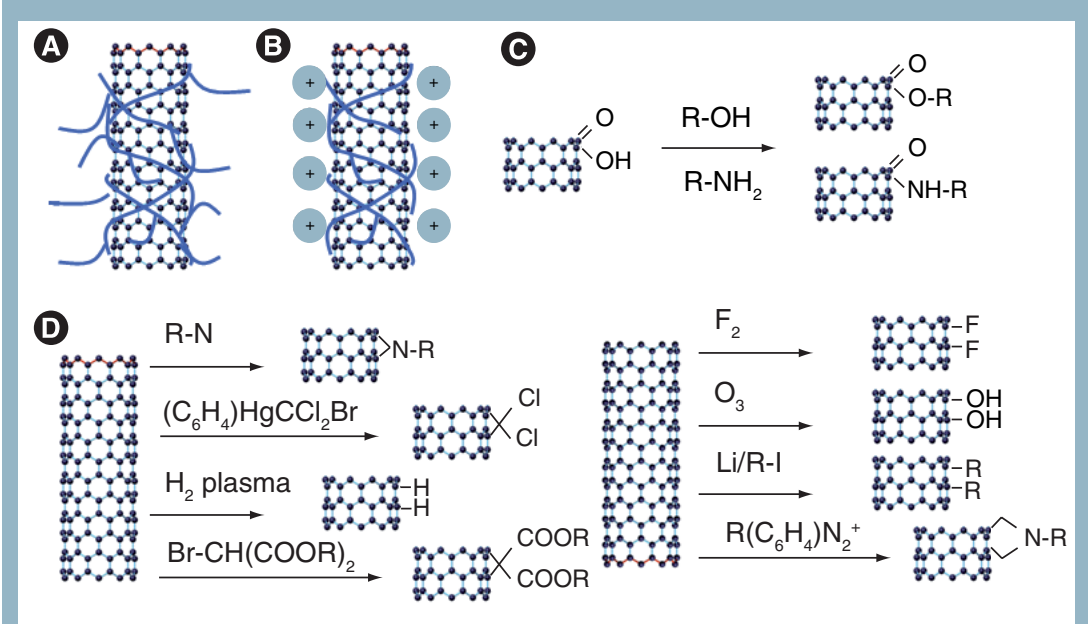

Figure 1. Strategies for nanotube dispersion. (A) Nanotubes are stably dispersed through steric stabilization by adsorbed macromolecules (e.g., surfactant and polymer) on to the walls of the carbon nanotubes. (B) Nanotubes are stably dispersed through electrostatic repulsion of the charged macromolecules (e.g., surfactant, polymer, protein and nucleic acid) absorbed on to the walls of the carbon nanotubes. (C) Nanotube functionalization through thermal oxidation, followed by subsequent esterification or amidization of the carboxyl groups. (D) Some addition reactions for the functionalization of the nanotube sidewall [46]

as tiny straight 'nano-needles' able to penetrate the cell membrane more efficiently than longer nanotubes (i.e., $>1 \mu \mathrm{m}$ length), which are often arranged in a 'ball' or bundled shape.

Our findings are also consistent with data reported by Kostarelos et al. [28] who studied the interaction between a wide variety of functionalized CNTs (f-CNTs) and living cells. SWCNTs and MWCNTs (300-1000 nm in length) were covalently functionalized with various types of small molecules: ammonium-functionalized CNTs, acetamido-functionalized CNTs, fluorescein isothiocyanate (FITC)-functionalized CNTs, CNTs bifunctionalized with ammonium groups and FITC, CNTs bifunctionalized with methotrexate and FITC, CNTs bifunctionalized with amphotericin B and FITC, and CNTs bifunctionalized with ammonium groups and FITC. The results reported by these authors indicate that cellular internalization of CNTs is shared by a wide range of cell types, some of which exhibit deficient phagocytosis (fibroblasts) or lack the machinery for endocytosis (fungi, yeast and bacteria), and even under conditions that prevent uptake of extracellular material by energy-dependent mechanisms, including endocytosis.

Similar results were reported by Pantarotto et al. who tested FITC-labeled SWCNTs and peptide-SWCNT conjugates (a small peptide corresponds to the amino acid sequence 384-394 of the Gs protein) [7]. The nanotube length in this study was in the range of 300 to $1000 \mathrm{~nm}$. 


\section{Endocytosis}

Shi Kam et al. reported energy-dependent endocytosis as the internalization mechanism used by SWCNTs to carry various types of proteins noncovalently and nonspecifically bound to nanotube sidewalls inside the cells [29]. The SWCNTs used were short (tens to hundreds of nanometers) individual tubes $(1.5 \mathrm{~nm}$ in diameter) or small bundles (up to $5 \mathrm{~nm}$ in diameter). The proteins investigated included streptavidin, staphylococcal protein A, bovine serum albumin and cytochrome c. Intracellular protein transport and uptake via nanotube carriage are also generic for various adherent and non-adherent mammalian cell lines, including HeLa, NIH-3T3 fibroblast, HL60 and Jurkats cells. In another study the group investigated the endocytosis pathway [11], which encompasses several subcategories, including phagocytosis, pinocytosis, and clathrin-dependent receptor-mediated and clathrin-independent endocytosis [14]. RME, which is the most common pathway, is also called clathrin-dependent endocytosis. These authors investigated the intracellular transport of protein-SWCNT and DNA-SWCNT conjugates as individual nanotubes $(50-200 \mathrm{~nm})$ and small bundles with lengths of less than $1 \mu \mathrm{m}$. Cellular incubation with these SWCNT conjugates was carried out at $4-8^{\circ} \mathrm{C}$ and with cells pretreated with $\mathrm{NaN}_{3}$ but the fluorescence levels from cells observed with confocal microscopy were low. This indicates endocytosis as the internalization mechanism for the uptake of SWCNT conjugates. The pretreatment of the cells with either sucrose (hypertonic treatment) or a $\mathrm{K}^{+}$-depleted medium drastically reduced the level of cellular uptake of SWCNTs, confirming the involvement of the clathrin pathway for endocytotic cellular uptake of SWCNTs (RME). Additionally, folate (FA)-SWCNT conjugates were internalized selectively by FA-overexpressing tumor cells by RME.

Jin et al. also confirmed that RME is the internalization mechanism of single dispersed DNAwrapped SWCNTs of less than $1 \mu \mathrm{m}$ in length [30]. Interestingly, these authors investigated the relation between the endocytosis rate and SWCNT size. It is well established that particle size is a crucial parameter in determining its interactions with cells [31]. Particles smaller than $70 \mathrm{~nm}$ are efficient in harnessing the endocytotic machinery of the cell, and the optimal particle radius is $25 \mathrm{~nm}$ both experimentally [32] and theoretically [33]. All existing models predict a threshold radius $\left(\mathrm{r}^{*} \sim 25 \mathrm{~nm}\right)$, below which nanoparticle uptake by $\mathrm{RME}$ is impossible. For nanoparticles of tubular shape, one needs to consider the effective radius. An effective radius of $25-70 \mathrm{~nm}$ corresponds to a nanotube length $l^{*}$ of $280-900 \mathrm{~nm}$ for SWCNTs and 120-600 $\mathrm{nm}$ for MWCNTs $\left(r^{*}=\mathrm{a} / \ln (2 \mathrm{a} / \mathrm{b})\right.$ with $a$ and $b$ major and minor semiaxes). The experimental data of Jin et al. demonstrated that the highest uptake occurs with nanotubes 320 $\pm 30 \mathrm{~nm}$ in length and decreases with longer $(660 \pm 40 \mathrm{~nm})$ and shorter $(130 \pm 18 \mathrm{~nm})$ nanotubes. To explain why SWCNTs shorter than $280 \mathrm{~nm}$ can be endocytosed in this manner, the authors proposed a model based on the clustering of the nanotube on the external cellular membrane. In this model, nanotubes first adsorb reversibly to the plasma membrane and form receptor-bound complexes capable of membrane surface diffusion. This process invariably causes clusters of sufficient size to lower the otherwise prohibitive thermodynamic barrier. The quantitative model proposed to correlate endocytosis rate with SWCNT size is in good agreement with the aforementioned experimental data.

Endocytotic uptake of the whole CNT-ssDNA complexes was also reported by Heller et al. who used ssDNA to achieve the solubilization of the CNTs in aqueous solutions. The authors demonstrated an endocytosis-dependent internalization with peri-nuclear localization [34]. RME is generally invoked as the internalization mechanism for CNTs functionalized with specific cell ligands. Bhirde et al. demonstrated that SWCNTs (110 $\pm 50 \mathrm{~nm}$ in length) functionalized with cisplatin and EGF can selectively and efficiently target squamous cancer cells that overexpress the EGF receptor as demonstrated by studies performed in vitro and on living mice. EGF receptor-targeted bioconjugates were much more efficient at killing cancer cells than untargeted controls containing the same drug, suggesting a major ligand receptormediated endocytosis pathway for cellular uptake both in vitro and in vivo [35]. Similarly, nanotube uptake via endocytosis has also been reported for SWCNTs functionalized with three different agents (doxorubicin, a monoclonal antibody and fluorescein) [36], for SWNTs functionalized with a FA moiety (selective internalization by cells labeled with FA receptor tumor markers) [37] and for SWCNTs functionalized with an integrin $\alpha_{v} \beta_{3}$ monoclonal antibody [38].

Finally the spectroscopic studies of Becker et al. have confirmed the importance of length on the cellular uptake of CNT-ssDNA complexes [39]. Their results indicated a length-selective cell uptake of nanotubes. Although length-dependent cellular uptake seems to be a general phenomenon, the exact threshold is expected to vary with cell 
type and the nature of the direct physical forces that govern attraction of the complexes to cells and which in turn influence the uptake process.

\section{A unified explanation}

The discrepancy in uptake mechanisms can be clarified by careful scrutiny of the physicochemical properties of the CNT complexes (TABLE 1).

It is known that phagocytosis is a process of engulfment by which macrophages ingest cellular fragments or microparticles. Above several hundred nanometers, the primary immune system (phagocytic cells and associated machinery) is primarily involved in clearance of these foreign particles. Phagocytosis is thus evoked as the main internalization pathway for CNT aggregates that are several micrometers in size [11] and for single dispersed CNT with a length of $1 \mu \mathrm{m}$ or more (Figure 2A1 \& B2) [8].

Strong endocytosis-dependent cellular internalization is involved when the CNTs form supramolecular complexes. This occurs when the CNT complexes (covalently or otherwise) proteins, oligonucleotides $[9,29,30,34]$ and specific cell ligands [37], including growth factors [35] and monoclonal antibodies $[36,38]$. In addition, more hydrophobic nanotubes should exhibit a similar behavior owing to the adsorption of extracellular proteins to the surfaces of nanotubes [40].
When protein adsorption occurs, the resultant outer protein coating becomes the most important parameter in determining the nanotube-cell interaction. Specifically, RME has been identified as the endocytosis pathway $[9,30]$. Theoretical and experimental studies have demonstrated that there is a size cutoff above which particles are unable to use this endocytotic route $(70 \mathrm{~nm}$ for spherical particle, corresponding to 900 and $600 \mathrm{~nm}$ for SWCNTs and MWCNTs, respectively). However, the exact threshold is expected to vary with cell type and the physical forces that attract complexes to cells. These observations can be explained by noting that RME is mediated by the tri-skeletal membrane protein clathrin that when activated, forms cage-like capsules that engulf the particle (or other foreign body) and draw it inside the cell, first to the primary endosomal cavity, and then to different locations depending on the nature of the particle. The molecular structure of clathrin is such that there is a natural size limit $(\sim 150 \mathrm{~nm})$ for these cages, and thereby a size cutoff above which particles are unable to use this endocytotic route. In support of this, it has been observed that only single dispersed sub-micron nanotubes are internalized via RME (FIgure 2C1). However, experimental data contradict the theory predicting a minimal length below which the uptake

\section{Table 1. Summary of the literature on carbon nanotube internalization mechanisms.}

\begin{tabular}{|c|c|c|c|c|c|}
\hline Type & Complex* & Dispersion status & Length/size & $\begin{array}{l}\text { Uptake } \\
\text { mechanism }\end{array}$ & Ref. \\
\hline SWCNT & - & Bundles & $>1 \mu \mathrm{m}$ & Phagocytosis & {$[11]$} \\
\hline SWCNT & Pluronic ${ }^{\circledR}$ F108:CNT & Single dispersed & $>1 \mu \mathrm{m}$ & Phagocytosis & {$[8]$} \\
\hline MWCNT & Pluronic F127:CNT & Single dispersed & $<1 \mu \mathrm{m}$ & Diffusion & [14] \\
\hline $\begin{array}{l}\text { SWCNT } \\
\text { MWCNT }\end{array}$ & $\begin{array}{l}\mathrm{NH}_{3}{ }^{+}-\mathrm{CNT}, \mathrm{NHCOCH}-\mathrm{CNT}, \mathrm{FITC}-\mathrm{CNT}, \\
\mathrm{NH}_{3}{ }^{+}-\mathrm{CNT}-\mathrm{FITC}, \mathrm{FITC}-\mathrm{CNT}-\mathrm{MTX}, \mathrm{AmB}-\mathrm{CNT}-\mathrm{FITC}, \\
\mathrm{NH}_{2}-\mathrm{CNT}-\mathrm{FITC}\end{array}$ & Single dispersed & $<1 \mu \mathrm{m}$ & Diffusion & {$[15]$} \\
\hline SWCNT & FITC-CNT, peptide-CNT & Single dispersed & $0.3-10 \mu \mathrm{m}$ & Diffusion & {$[7]$} \\
\hline SWCNT & SA-CNT, SpA-CNT, BSA-CNT, cytc-CNT & $\begin{array}{l}\text { Single dispersed/ } \\
\text { bundles }\end{array}$ & $0.01-0.1 \mu \mathrm{m} /<5 \mathrm{~nm}$ & Endocytosis & [29] \\
\hline SWCNT & DNA:CNT, BSA:CNT, SA:CNT & $\begin{array}{l}\text { Single dispersed/ } \\
\text { bundles }\end{array}$ & $\begin{array}{l}0.05- \\
0.2 \mu \mathrm{m}) /<1 \mu \mathrm{m}\end{array}$ & RME & [9] \\
\hline SWCNT & DNA:CNT & Single dispersed & $<1 \mu \mathrm{m}$ & RME & [30] \\
\hline SWCNT & ssDNA:CNT & Single dispersed & - & endocytosis & [34] \\
\hline SWCNT & EGF-CNT-cisplatinum & Single dispersed & $110 \pm 50 \mathrm{~nm}$ & RME & {$[35]$} \\
\hline SWCNT & DOX:CNT-BSA-(fluorescein, monoclonal antibody) & $\begin{array}{l}\text { Single dispersed/ } \\
\text { bundles }\end{array}$ & $0.2-1 \mu \mathrm{m} /<4 \mathrm{~nm}$ & RME & [36] \\
\hline SWCNT & $\begin{array}{l}\text { CNTS:DNA-Cy3 } \\
\text { CNTs:PL-PEG--FA } \\
\text { PL-PEG-FA:CNT:PL-PEG-FITC }\end{array}$ & $\begin{array}{l}\text { Single dispersed/ } \\
\text { bundles }\end{array}$ & $0.2-1 \mu \mathrm{m} /<4 \mathrm{~nm}$ & RME & [37] \\
\hline SWCNT & CNT-PEG-SpA: $\alpha v \beta 3-F I T C$ & Single dispersed & $0.5-1 \mu \mathrm{m}$ & RME & {$[38]$} \\
\hline
\end{tabular}




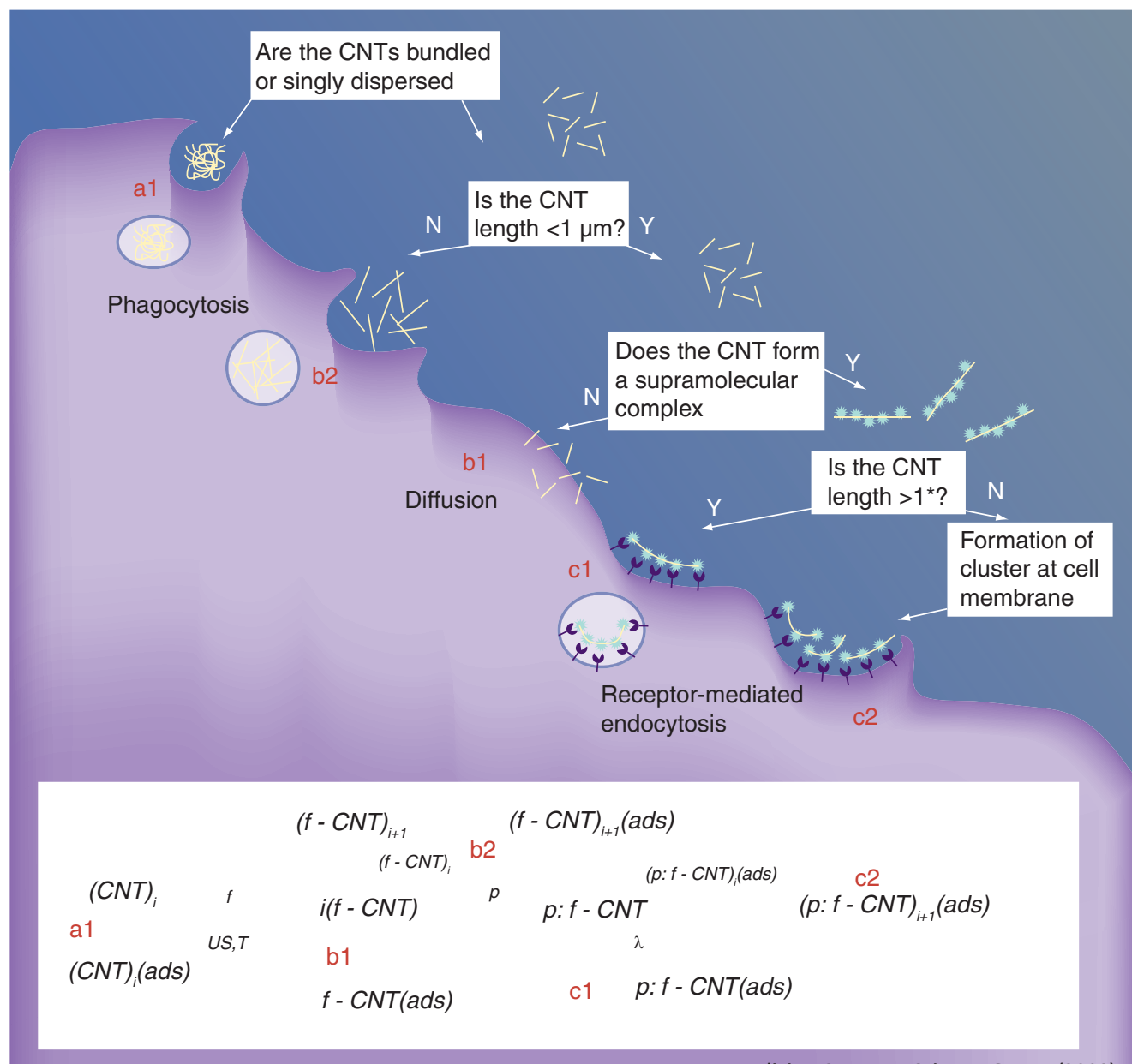

Nanomedicine (c) Future Science Group (2009)

Figure 2. Parameters influencing the carbon nanotube internalization mechanisms.

Inset: Nanotube bundles $(C N T)_{i^{\prime}}$, functionalized with (macro)molecules $(f)$ with the aid of sonication (US) and/or temperature (T)), are dispersed in single nanotubes $f-C N T$; this $f$ - CNT can lead to the formation of supramolecular structure $p: f-C N T$. (A1) $(C N T)_{i}$ interact with cell membrane and are internalized via phagocytosis; (B1) $f$ - CNT enter the cells by diffusion; (B2) nanotube dispersion is not stable and nanotubes cluster in solution (nanotube clusters enter the cell by phagocytosis);

(C1) $p: f-C N T$ enters the cell by ligand-receptor ( $\lambda$ ) binding; (C2) $p: f-C N T$ cluster on cell membrane and enter by receptor-mediated endocytosis.

CNT: Carbon nanotube; f-CNT: Functionalized CNT.

by RME becomes thermodynamically impossible. To explain this, Jin et al. proposed a model in which CNTs below this critical length form clusters with a radius of sufficient size that eventually satisfy the thermodynamic requirements for endocytosis (Figure 2C2) [30].

The internalization of supramolecular complexes via RME is well established and demonstrated for supramolecular complexes formed between biological macromolecules and other nanoparticles, such as liposome-oligodeoxynucleotide, polymer-DNA, cation lipid-DNA [41], polymeric core-shell architectures [42].

Diffusion mechanisms are invoked for functionalized nanotubes, which prevent protein adsorption and the formation of supramolecular complexes. The internalization process entails insertion and diffusion of nanotubes across cell membranes (Figure 2B1) but the exact mechanism of this process remains unclear. Pantarotto et al. [7] suggest that CNTs may behave like cell penetrating peptides and related synthetic oligomers [43]. The penetration of the nanotubes through the plasma membrane akin to a 'nano-syringe' has also been theoretically demonstrated [44]. Molecular dynamics simulations demonstrate that a generic hydrophobic nanotube with hydrophilic functionality at its terminals spontaneously inserts into, aligns and carries molecules across the cell membrane by a lipid-assisted mechanism. This simulation suggests that the uptake of the nanotube could be the result of amphipathic properties of both the cellular membrane and f-CNTs. It has also been 


\section{Table 2. Internalization mechanisms for the reactions of Figure 2.}

\begin{tabular}{|lllr}
\hline Pathway & Main internalization mechanism & Favored by & Ref. \\
\hline A1 & Phagocytosis & Nanotube bundles, single dispersed CNT with length $>1 \mu \mathrm{m}$ & {$[6,9]$} \\
\hline B1 & Passive diffusion & CNT single dispersed, length $<1 \mu \mathrm{m}$ & {$[5,12,13]$} \\
B2 & Phagocytosis & Nanotube clusters & {$[9]$} \\
C1 & RME & Supramolecular complex, $r>r^{*}$ & {$[7,26,27,31,32,33]$} \\
C2 & RME & Supramolecular complex, $r<r^{*}$ \\
\hline CNT: Carbon nanotube; RME: Receptor-mediated endocytosis. & & {$[27]$} \\
\hline
\end{tabular}

reported that the CNT length influences the penetration capability: shorter nanotubes (i.e., hundreds of nanometers in length) are able to enter cell membrane acting as tiny needles [26] more efficiently than longer nanotubes (i.e., several micrometers in length), which are arranged in a bundled configuration. It is important to note that the hypothesis of diffusion process is supported by direct imaging $[11,45]$. Specifically Cheng et al. applied a combination of advanced microscopy techniques to image carbon nanoparticles within cells [45]. They used energyfiltered TEM, high angle annular dark field scanning TEM, tomography and confocal microscopy to generate 3D images enabling determination of nanoparticle spatial distribution in a cell. Some SWCNTs and MWCNTs were observed translocating the plasma, lysosomal and nuclear membranes supporting the nano-needle hypothesis.

\section{Future perspective}

This article has sought to explain the entry mechanisms that govern the cellular internalization of CNTs and their cargos. These are influenced by a few parameters, which are summarized in Figure 2 \& Table 2 . This schema should be useful to nano-scientists interested in 'design criteria' for the development of CNT-based carrier systems for cell therapy.
However, future research is required to fill the many gaps in our knowledge of the cellular internalization mechanisms of CNTs. Currently, several fundamental issues remain unresolved at the biomolecular level, especially with regard to internalization pathways and intracellular traffic. An ideal approach would combine molecular biology assays with nanometer scale imaging to elucidate the detailed physiological mechanisms and structural effects of CNT internalization. Full understanding of these issues will enable the rational design of CNT-based nano-pharmaceuticals in the near future. The appropriate cell internalization pathway selected will depend on the medical application (e.g., drug delivery, cancer therapy and gene therapy) and on the exact intracellular target (e.g., cytoplasm and nucleus).

\section{Financial \& competing interests disclosure}

This work has been performed in the framework of the Non Invasive Nanotransducer for In vivo Gene Therapy (NINIVE) project funded by the European Commission (Contract No. 033378). The authors have no other relevant affiliations or financial involvement with any organization or entity with a financial interest in or financial conflict with the subject matter or materials discussed in the manuscript apart from those disclosed.

No writing assistance was utilized in the production of this manuscript.

\section{Executive summary}

- Carbon nanotubes (CNTs) can be used as excellent intracellular transporters for drug delivery provided they are made water soluble by dispersion, since this is essential for biocompatibility.

- The main methods for CNT dispersion are: surfactant-assisted dispersion, functionalization of CNT sidewalls and biomolecular dispersion.

- Understanding the internalization mechanisms is crucial for pharmaceutical application of CNTs.

- There are three crucial parameters influencing CNT interactions with cells:

- The degree of dispersion - depending on the dispersion process the nanotubes can be in the form of bundles, clusters or single dispersed CNTs.

- The formation of supramolecular complexes - this occurs when the nanotubes are functionalized with proteins and nucleic acids or when the polymer coating leads to the adsorption of proteins from extracellular matrix.

- Nanotube length.

- The internalization process can occur by phagocytsis, endocytocis or diffusion.

- Phagocytosis is the internalization pathway for CNT aggregates, bundles, cluster or single dispersed nanotubes that are $1 \mu \mathrm{m}$ or more in length. Endocytosis is the internalization mechanism for nanotubes that form supramolecular structures. Otherwise, the internalization mechanism is by diffusion. 


\section{Bibliography}

Papers of special note have been highlighted as:

- of interest

"I of considerable interest

1 Iijima S: Helical microtubules of graphitic carbon, Nature 354, 56-58 (1991).

2 Saito R, Dresselhaus G, Dresselhaus MS: In: Physical Properties of Carbon Nanotubes. Imperial College Press, London, UK 1-29 (1998).

3 Lacerda L, Raffa V, Prato M, Bianco A, Kostarelos K: Cell-penetrating carbon nanotubes in the delivery of therapeutics. Nano Today 2, 38-43 (2007).

4 Foldvari M, Bagonluri M: Carbon nanotubes as functional excipients for nanomedicines: II. Drug delivery and biocompatibility issues. Nanomedicine 4, 183-200 (2008).

5 Pastorin G: Crucial functionalizations of carbon nanotubes for improved drug delivery: a valuable option? Pharm. Res. 26, 746-769 (2009).

6 Firme CP, Bandaru PR: Toxicity issues in the application of carbon nanotubes to biological systems. Nanomedicine doi:10.1016/j. nano.2009.07.003 (2009) (Epub ahead of print).

7 Pantarotto D, Briand JP, Prato M, Bianco A: Translocation of bioactive peptides across cell membranes by carbon nanotubes. Chem. Commun. 1, 16-17 (2004).

\# Highlights for the first time that carbon nanotube uptake can occur with an energy-independent nonendocytotic pathway.

8 Cherukuri P, Bachilo SM, Litovsky SH, Weisman RB: Near-infrared fluorescence microscopy of single-walled carbon nanotubes in phagocytic cells. J. Am. Chem. Soc. 126, 15638-15639 (2004).

9 Shi Kam NW, Liu Z, Dai H: Carbon nanotubes as intracellular transporters for proteins and DNA: an investigation of the uptake mechanism and pathway. Angew. Chem. Int. 45, 577-581 (2006).

=IInvestigates the intracellular transport mechanisms of protein-single wall carbon nanotube (SWCNT) and DNA-SWCNT conjugates and demonstrates that clathrindependent endocytosis is the pathway of nanotube internalization.

10 Homma Y, Takagi D, Suzuki S, Kanzaki KI, Kobayashi Y: Electron-microscopic imaging of single-walled carbon nanotubes grown on silicon and silicon oxide substrates. J. Electron. Microsc. 54, 3-7 (2005).

11 Porter AE, Gass M, Muller K, Skepper JN, Midgley PA, Welland M: Direct imaging of single-walled carbon nanotubes in cells. Nat. Nanotechnol. 2, 713 (2007).
12 Brown G, Bailey SR, Novotny Met al.: High yield incorporation and washing properties of halides incorporated into single walled carbon nanotubes. Appl. Phys. 76, 457-462 (2003).

13 Hong H, Gao T, Cai W: Molecular imaging with single-walled carbon nanotubes. Nano Today 4, 252-261 (2009).

14 Tasis D, Tagmatarchis N, Bianco A, Prato M: Chemistry of carbon nanotubes. Chem. Rev. 6, 1105-1136 (2006)

15 Foldvari M, Bagonluri M: Carbon nanotubes as functional excipients for nanomedicines: I. Pharmaceutical properties. Nanomedicine 4, 73-78 (2008).

16 Yang DQ, Rochette JF, Sacher E: Functionalization of multiwalled carbon nanotubes by mild aqueous sonication. J. Phys. Chem. B. 109(16), 7788-7794 (2005).

17 Ham HT, Choi YS, Chung IJ: An explanation of dispersion states of single-walled carbon nanotubes in solvents and aqueous surfactant solutions using solubility parameters. J. Colloid Interface Sci. 286, 216-223 (2005).

18 Vaisman L,Wagner HD, Marom G: The role of surfactants in dispersion of carbon nanotubes. Adv. Colloid Interface Sci. 128-130, 37-46 (2006).

19 Balavoine F, Schultz P, Richard C, Mallouh V, Ebbesen W, Mioskowski C: Helical crystallization of proteins on carbon nanotubes: a first step towards the development of new biosensors. Angew. Chem. Int. Ed. 38, 1912 (1999).

20 Bradley K, Briman M, Star A, Gruner G: Charge transfer from adsorbed proteins. Nano Lett. 4, 253 (2004).

21 Tzeng Y, Huang TS, Chen YC, Liu C, Liu YK: Hydration properties of carbon nanotubes and their effects on electrical and biosensor applications. N. Diamond Front. Carbon Technol. 14, 193 (2004).

22 Singh R, Pantarotto D, McCarthy D et al.: Binding and condensation of plasmid DNA onto functionalized carbon nanotubes: toward the construction of nanotube-based gene delivery vectors. J. Am. Chem. Soc. 127, 4388-4396, (2004).

23 Liu Y, Wu DC, Zhang WD et al: Polyethylenimine-grafted multiwalled carbon nanotubes for secure noncovalent immobilization and efficient delivery of DNA. Ang. Chem. Int. Ed. 44, 4782-4785 (2005)

24 Baker SE, Cai W, Lasseter TL, Weidkamp KP, Hamers RJ: Covalently bonded adducts of deoxyribonucleic acid (DNA) oligonucleotides with single-wall carbon nanotubes: synthesis and hybridization. Nano Lett. 2, 1413-1417 (2002).
25 Dwyer C, Guthold M, Falvo M, Washburn S, Superfine R, Erie D: DNA functionalized single-walled carbon nanotubes. Nanotechnology 13, 601-604 (2002).

26 Raffa V, Ciofani G, Nitodas S, Karachalios T: Can the properties of carbon nanotubes influence their internalization by living cells? Carbon 46, 1600 (2008).

27 Mukherjee S, Ghosh RN, Maxfield FR: Endocytosis. Physiol. Rev. 77, 759-803 (1997).

28 Kostarelos K, Lacerda L, Pastorin G et al.: A cellular uptake of functionalized carbon nanotubes is independent of functional group and cell type. Nat. Nanotech. 2, 108-13 (2007).

29 Shi Kam NW, Dai H: Carbon nanotubes as intracellular protein transporters: generality and biological functionality. J. Am. Chem. Soc. 127, 6021 (2005).

30 Jin H, Heller DA, Sharma R, Strano MS: Size-dependent cellular uptake and expulsion of single-walled carbon nanotubes: single particle tracking and a generic uptake model for nanoparticles. ACS Nano 3, 149-158 (2009).

- The authors measure the cellular uptake and expulsion rates of length-fractionated SWCNTs and develop a quantitative model to correlate endocytosis rate with nanoparticle size.

31 Jiang W, Kim BYS, Rutka JT, Chan WCW: Nanoparticle-mediated cellular response is size-dependent. Nat. Nanotechnol. 3, 145-150 (2008).

32 Nakai T, Kanamori T, Sando S, Aoyama Y: Remarkably size-regulated cell invasion by artificial viruses. Saccharide-dependent self-aggregation of glycoviruses and its consequences in glycoviral gene delivery. J. Am. Chem. Soc. 125, 8465-8475 (2003).

33 Gao H, Shi W, Freund LB: Mechanics of receptor-mediated endocytosis. Proc. Natl Acad. Sci. USA 102, 9469-9474 (2005).

34 Heller DA, Baik S, Eurell TE, Strano MS: Single-walled carbon nanotube spectroscopy in live cells: towards long-term labels and optical sensors. Adv. Mater. 17, 2793-2799 (2005).

35 Bhirde AA, Patel V, Gavard J et al.: Targeted killing of cancer cells in vivo and in vitro with EGF-directed carbon nanotube-based drug delivery. ACS Nano 3, 307-316 (2009).

36 Heister E, Neves V, Tilmaciu C et al.: Triple functionalisation of single-walled carbon nanotubes with doxorubicin, a monoclonal antibody, and a fluorescent marker for targeted cancer therapy. Carbon 47 , 2152-2160 (2009). 
37 Shi Kam NW, O’Connell M, Wisdom JA, Dai H: Carbon nanotubes as multifunctional biological transporters and near-infrared agents for selective cancer cell destruction. Proc. Natl Acad. Sci. USA 102, 11600-11605 (2005).

38 Ou Z, Wu B, Xing D, Zhou F, Wang H, Tang Y: Functional single-walled carbon nanotubes based on an integrin $\alpha_{v} \beta_{3}$ monoclonal antibody for highly efficient cancer cell targeting. Nanotechnology 20 , 105102 (2009).

39 Becker ML, Fagan JA, Gallant ND et al: : Length-dependent uptake of DNA-wrapped single-walled carbon nanotubes. Adv. Mater. 19, 939-945 (2007)
40 Lynch I: Are there generic mechanisms governing interactions between nanoparticles and cells? Epitope mapping the outer layer of the protein. Mater. Interface Phys. 373, 511-520 (2007).

41 Wang Y, Shen J: Progress in non-viral gene delivery systems fabricated via supramolecular assembly. Chin. Sci. Bull. 50, 289-294 (2005).

42 Haag R: Supramolecular drug-delivery systems based on polymeric core-shell architectures. Angew. Chem. Int. Ed. 43, 278-282 (2004).

43 Alves ID, Correia I, Jiao CY et al:: The interaction of cell-penetrating peptides with lipid model systems and subsequent lipid reorganization: thermodynamic and structural characterization. J. Pept. Sci. 5 , 200-209 (2009).
44 Lopez CF, Nielsen SO, Moore PB, Klein ML: Understanding nature's design for a nanosyringe. Proc. Natl Acad. Sci. USA 101, 4431-4434 (2004).

- Theoretically demonstrates that the nanotubes can penetrate through the plasma membrane acting as tiny needles.

45 Cheng C, Porter AE, Muller K et al.: Imaging carbon nanoparticles and related cytotoxicity. J. Phys. Conf. Ser. 151, 012030 (2009).

- SWCNTs and multiwall carbon nanotubes were observed translocating the plasma, lysosomal and nuclear membranes, therefore supporting the nano-needle hypothesis.

46 Burghard M, Balasubramanian K: Chemically functionalized carbon nanotubes. Small 2, 180 -192 (2005). 